Akt. Urol. 17 (1986) $177-180$

(C) Georg Thieme Verlag Stuttgart · New York

\section{Rationelle Diagnostik der erektilen Dysfunktion unter Anwendung eines pharmakologischen Testes}

\author{
W. Bähren, Ch.Stief, W.Scherb, H. Gall, A. Gallwitz, J. Altwein
}

Radiologische Abteilung (Ltd. Arzt: Oberstarzt Dr. W. Wierschin)

Urologische Abteilung (Kommissarisch Ltd. Arzt: Oberstabsarzt Dr. W. Thon)

Neurologische Abteilung (Ltd. Arzt: Oberstarzt Priv.-Doz. Dr. Kriebel)

Dermatologische Abteilung (Ltd. Arzt: Oberstarzt Prof. Dr. O. E. Rodermund)

Urologische Abteilung Krankenhaus Barmherzige Brüder München (Ltd. Ärzte:

Prof. Dr. J. E. Altwein, Dr. Schneider)

\section{Einleitung}

Bei der nicht-invasiven Diagnostik der erektilen Dysfunktion ist von entscheidender therapeutischer Relevanz, ob eine zuverlässige Differenzierung zwischen vaskulär und nicht-vaskulär bedingter Erektionsstörung gelingt.

Zur Beantwortung dieser diagnostischen Schlüsselfrage wurden in den letzten 10 Jahren zahlreiche nicht-invasive und invasive Testmethoden und Untersuchungsverfahren entwickelt, standardisiert und an mehreren Zentren weltweit meist in Form eines multidisziplinären Abklärungsprogrammes angewandt.

Eine rationelle und intelligente Lösung des diagnostischen Problems erektiler Dysfunktion muß aus der Vielzahl verfügbarer Methoden diejenigen auswählen, die unter Abwägung folgender Gesichtspunkte zu einer therapeutisch orientierten Entscheidung führen:

- Verfügbarkeit der Methode (availability)

- Objektivität (objectivity)

- Genauigkeit (accuracy)

- Einfachheit (simplicyty)

- Zeitaufwand (amount of time)

- Kostenaufwand (amount of costs).

In der vorliegenden Studie soll untersucht werden, ob mit Hilfe der diagnostischen Anwendung intrakavernös applizierter vasoaktiver Substanzen an einem multidisziplinär untersuchten (Doppler, PBI, BCR, MMPI, Kavernosographie, selektive Arteriographie) Kollektiv impotenter Männer eine Straffung und Optimierung des aufwendigen diagnostischen Ablaufs möglich ist.

\section{Material und Methode}

In der Zeit von Januar 1985 bis Januar 1986 wurden 135 Patienten im Alter von 19 bis 65 Jahren wegen einer mindestens $1 \mathrm{Jahr}$ bestehenden erektilen Dysfunktion multidisziplinär untersucht. Der Untersuchungsgang bei allen Patienten gliederte sich in:

1. Erhebung der Anamnese und Sexualanamnese unter Einschluß eines psychosexuellen Fragebogens,

2. körperliche Untersuchung,

3. Erhebung der Laborparameter ( $\mathrm{Hb}, \mathrm{Hk}$, Erythrozyten, Leukozyten, GOT, GPT, Gamma-GT, alkalische Phosphatase,

\section{Zusammenfassung}

Bei 100 multidisziplinär untersuchten Patienten mit mindestens 1jähriger erektiler Dysfunktion (E. D.) wurde zu diagnostischen Zwecken ein pharmakologisches Testverfahren mit intrakavernöser Applikation eines PapaverinPhetolamingemisches angewandt. Aufgrund dieses pharmakologischen Testes gelingt eine Einordnung in 1. nichtvaskulär bedingte E. D., 2. vaskuläre E. D. mit pathologischen inflow-Bedingungen und 3. vaskuläre E. D. mit pathologischen outflow-Bedingungen. Gruppe I ( $\mathrm{n}=13$ ) erreichte mit durchschnittlich $0,43 \mathrm{ml}$ unserer SKAT-Lösung eine Erektion mit mittlerer Rigidität, Gruppe II ( $\mathrm{n}=68$ ) benötigte durchschnittlich $1,05 \mathrm{ml}$. Bei 13 von 19 Patienten der Gruppe III kam es nach intrakavernöser Gabe von $3 \mathrm{ml}$ nicht zu einer Erektion, die übrigen 6 Patienten mit pathologischen Ausflußbedingungen erzielten mit $3 \mathrm{ml}$ eine für Kohabitation noch ausreichende Erektion.

Mit Hilfe des pharmakologischen Testes unter Anwendung vasoaktiver Substanzen läßt sich rationell eine therapierelevante Differenzierung zwischen vaskulärer und nicht-vaskulärer E. D. herbeiführen. Das aufwendige Untersuchungsprogramm kann dadurch gestrafft und optimiert werden.

Rational Diagnosis of Erectile Dysfunction by Using a Pharmacological Test

100 multidisciplinary evaluated patients with erectile dysfunction of more than one year duration were investigated by a pharmacological test using intracavernously applicated mixture of papaverine and phentolamine.

The results showed, that differentiation between nonvascular origin (groupe $I, n=13$ ), vascular origin with pathological inflow (groupe II, $\mathrm{n}=68$ ) and vascular origin with pathological outflow (groupe III, $n=19$ ) correlated well with the multidisciplinary findings.

Groupe I achieved full erection with an average of $0,43 \mathrm{ml}$, groupe II with an average of $1,05 \mathrm{ml}$ of our papaverin-phentolamine-solution. 13 of 19 patients (groupe III) with pathological outflow condition did not achieve erection with $3 \mathrm{ml}$ testdosis. The remainding 6 patients of groupe III showed full tumescence but insufficient rigidity.

The pharmacological test of erectile dysfunction is useful in the differential diagnosis of non-vascular and vascular origin and leads to decisions with relevant therapeutic impact.

The diagnostic examination of patients with erectile dysfunction can be shortened and improved by pharmacological testing.

Key-Words: Erectile dysfunction - Doppler ultrasound - Arteriography - Phentolamine - Papaverine

Bilirubin, Kreatinin, Harnsäure, Cholesterin, Triglyceride, Urinstatus, Sediment),

4. Erhebung der Hormonparameter (Serum-Testosteron und Serum-Prolaktin), 
5. psychologisches Interview und Psychometrie (MMPI, FPI),

6. BCR-Latenzzeitmessung,

7. penile Doppler-Ultraschalluntersuchung aller 4 Penisgefäße proximal und distal,

8. Bestimmung des penil-brachialen Blutdruckindexes (PBI).

Die nicht-invasiven Untersuchungen fanden unter ambulanten Bedingungen räumlich und zeitlich koordiniert statt. Bei 100 Patienten erfolgte im Anschluß an die Untersuchungen die diagnostische Applikation eines Gemisches aus Papaverinhydrochlorid und Phentolaminmesylat in einem Mischungsverhältnis von $15 \mathrm{mg}$ : 0,5 mg in $1 \mathrm{ml}$ physiologischer Kochsalzlösung. $\mathrm{Ab}$ September 1985 wurde die diagnostische intrakavernöse Injektion von Papaverin/Phentolamin-Gemisch in einer Dosierung von $7,5 \mathrm{mg} / 0,25 \mathrm{mg}$ Bestandteil der ambulanten diagnostischen Phase. 19 Patienten mit fehlender oder unzureichender erektiler Antwort auf eine Testdosis von maximal $3 \mathrm{ml}$ wurden mit der dynamischen Infusionskavernosographie im Hinblik auf eine venöse Insuffizienz untersucht. Sofern aufgrund eines pathologischen Doppler-Befundes eine alleinige vaskuläre erektile Dysfunktion vermutet wurde und eine Revaskularisation ins Auge gefaßt wurde, erfolgte die selektive Arteriographie der pudendal-penilen Gefäße unter Anwendung intrakavernös applizierten Papaverin/Phentolamin-Gemisches. Dies war bei 49 Patienten mit posttraumatischer erektiler Dysfunktion nach Becken- $/$ Penistrauma, primärer erektiler Dysfunktion und sekundärer erektiler Dysfunktion bei Verdacht auf penile Gefäßdysplasie der Fall. Das Durchschnittsalter der 49 im obengenannten Zeitraum arteriographierten Patienten lag bei 40,4 Jahren.

Kontraindikationen der diagnostischen Testung waren: schwere koronare Herzkrankheit, Herzrhythmusstörung, Niereninsuffizienz, Leberinsuffizienz, Herzinsuffizienz, manifeste Suchterkrankung, arterielle Verschlußkrankheit im Stadium III und IV, Störung der kognitiven und geistigen Entwicklung.

Vor der pharmakologischen Testung erfolgte eine ausführliche Aufklärung über Wesen und Umfang des Testes sowie über alle denkbaren möglichen Risiken (Infektion, Blutung, Fibrose, Kreislaufreaktion, Unverträglichkeit).

In jedem Fall lag eine schriftliche Einwilligungserklärung vor.

Die Injektion von beginnend $0,5 \mathrm{ml}$ stufenweise bis maximal $3,0 \mathrm{ml}$ der Lösung erfolgte dorsal an der Peniswurzel ca. 3-4 mm lateral der Penismitte in das Zentrum des rechten oder linken Corpus cavernosum. Im ersten Halbjahr wurde eine Injektionsnadel der Stärke Nr. 23, seit Juli 85 der Stärke Nr. 26 verwendet. Nach der Injektion wurde die Zeit bis zum Eintritt der erektilen Antwort und die Qualität der Antwort optisch und palpatorisch registriert. Die maximale Antwort wurde in $6 \mathrm{Stu}-$ fen klassifiziert:

$\mathrm{E}-0=$ keine Reaktion

$\mathrm{E}-1=$ geringe Tumeszenz

$\mathrm{E}-2=$ mittlere Tumeszenz

$\mathrm{E}-3=$ volle Tumeszenz

E $-4=$ volle Tumeszenz, mittlere Rigidität

$\mathrm{E}-5=$ volle Tumeszenz und volle Rigidität $=$ komplette Erektion.

Die Dauer der Tumeszenz/Rigiditätsphase wurde vermerkt. Bei 40 Patienten erfolgte ein fortlaufendes Monitoring von Herzfrequenz, systolischem und diastolischem Blutdruck über einen Zeitraum von 45 Minuten nach Injektion.

87 Patienten mit E 4 und E 5 wurden als Responder in das Therapieprogramm SKAT übernommen.

\section{Ergebnisse}

Bei 8 Patienten traten nach intrakavernöser Injektion der SKATLösung prolongierte Erektionen zwischen 6 und 10 Stunden auf. Bei 3 Patienten war diese Nebenwirkung durch Punktion des Corpus cavernosum und Ableiten von ca. $300 \mathrm{ml}$ Blut leicht und ohne Folgeschaden zu beherrschen. In den übrigen 5 Fällen konnte durch intrakavernöse Injektion eines Alpha-Mimetikums (Metaraminol, Araminum ${ }^{\circledR}$ von MSD Sharpe Dome) in einer Dosis von $2 \mathrm{mg}$ innerhalb von 10 Minuten eine vollständige Detumeszenz erreicht werden. Bei 16 weiteren Patienten wurde eine medikamentös verursachte Erektion nach 1 Stunde erfolgreich mit Metaraminol unterbrochen. Systemische oder lokale unerwünschte Wirkungen wurden dabei nicht beobachtet. Hämatome, Fibrosierung oder Entzündungen traten bisher weder nach SKAT-Test noch nach Therapie auf. Signifikante Auswirkungen (Friedman-Test) auf diastolischen und systolischen Blutdruck sowie auf die Herzfrequenz waren im Rahmen der kontinuierlichen Herz-Kreislaufüberwachung nicht nachzuweisen.

Aufgrund der Untersuchungsergebnisse des multidisziplinären Abklärungsprogramms wurden die Patienten hinsichtlich ihrer Ursache in 3 Gruppen unterteilt:

1. nicht-vaskulär (z.B. psychogen, neurogen),

2. vaskulär mit pathologischen inflow-Bedingungen (z.B. isoliert arteriell, gemischt arteriell und neurogen),

3. vaskulär mit pathologischen outflow-Bedingungen (z. B. isoliert venös, gemischt venös und arteriell).

13 Patienten mit nachgewiesener isoliert psychogener oder neurogener erektiler Dysfunktion (Gruppe 1) reagierten auf die intrakavernöse Injektion von durchschnittlich $0,43 \mathrm{ml}$ mit einer E 4 oder E 5 von mindestens 30 Minuten Dauer. 68 Patienten mit dopplersonographisch oder arteriographisch gesicherten pathologischen inflow-Bedingungen (Gruppe 2) erreichten mit einer Dosis zwischen 0,4 und $3 \mathrm{ml}$ eine $\mathrm{E} 4$ oder $\mathrm{E} 5$. Die durchschnittliche Dosis lag bei 1,05 ml SKAT-Lösung. Je ausgeprägter die pathologischen inflow-Bedingungen vorzufinden waren, um so höher war die erforderliche Dosis zur Erzielung einer E 4/5.

Bei 13 von 19 Patienten der Gruppe 3 war eine E 4/5 durch intrakavernöse Injektion vasoaktiver Substanzen von bis zu $3 \mathrm{ml}$ nicht zu erzielen. Bei allen Patienten dieser Gruppe konnte durch die dynamische Infusionskavernosographie ein pathologischer Maintaining-Index nachgewiesen werden. Es fanden sich dabei entweder Fistelbildungen zwischen Corpus cavernosum und Corpus spongiosum oder pathologisch drainierende Venen.

6 Patienten mit kavernosographisch nachgewiesenem pathologischen Abstrom in geringer Ausprägung erzielten mit $3 \mathrm{ml}$ eine für Kohabitation ausreichende Erektion.

\section{Diskussion}

Die Gruppe der Patienten, die wegen erektiler Dysfunktion ärztliche Hilfe sucht, ist nach Alter, Manifestationsdauer, Ätiologie, Grunderkrankung, Leidensdruck und Motivation sehr heterogen. Wegen der verschiedenen therapeutischen Optionen, die nur bei genauer Kenntnis der individuellen Ätiologie in angemessener Weise zum Einsatz kommen können, muß die Diagnostik in Form eines Stufenplanes mit hoher Effizienz in der Lage sein, zwischen den verschiedenen Ursachen der Erektionsstörung zu differenzieren (Abb. 1).

Aus der Vielzahl unterschiedlicher Untersuchungsmethoden muß zur rationellen therapieorientierten Diagnostik der E. D. eine Auswahl erfolgen, die effizient zwischen I. nicht-vaskulär, 


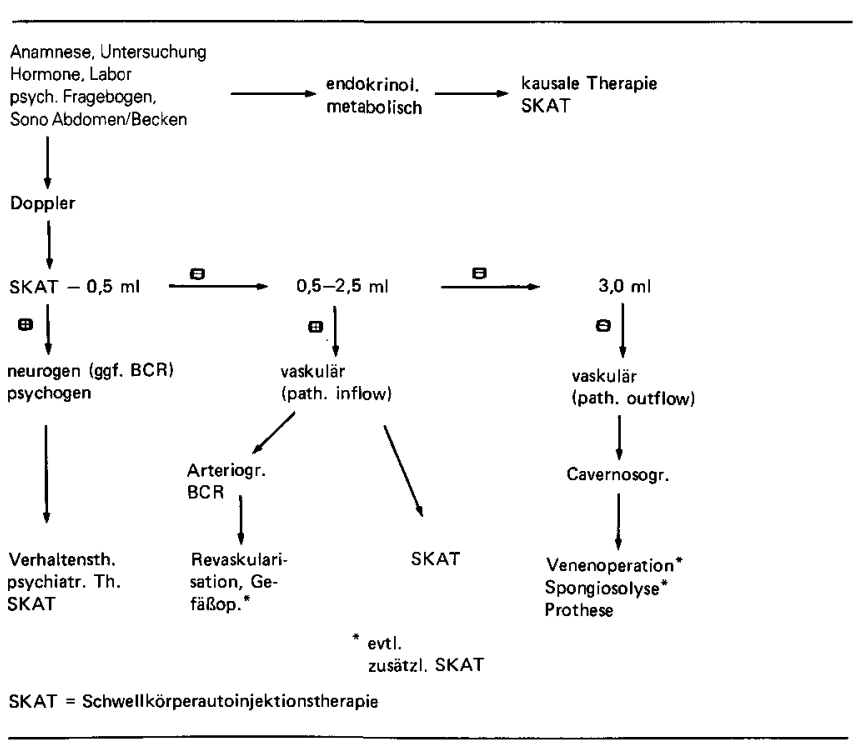

Abb. 1 Flußdiagramm: Diagnostik und Therapie der E. D.

II. vaskulär mit pathologischen inflow-Bedingungen und III. vaskulär mit pathologischen outflow-Bedingungen zu unterscheiden vermag.

Basis der Diagnostik ist und bleibt die ausführliche Anamnese im Hinblick auf die Sexualanamnese ergänzt durch einen psychosexuellen Fragebogen - sowie die körperliche Untersuchung. Psychologisches Interview und Psychometrie sollten, wenn möglich, in den Untersuchungsablauf integriert sein, weil dadurch das Gesamtverständnis des Patienten in seiner seelischen und sozialen Situation wesentlich verbessert werden kann. Aufklärung und Beratung an dieser Stelle können in einzelnen Fällen schon eine weitere Therapie überflüssig machen. Labor- und Hormonanalysen zur Erkennung von Risikofaktoren, Erkrankungen anderer Organsysteme oder hormonell bedingter erektiler Dysfunktion sind und bleiben obligat.

Die ursprüngliche Vorstellung, daß mit Hilfe der NPT-Messung (Nocturnale penile Tumeszenz) zwischen organischer und psychogener erektiler Dysfunktion mit hoher Sensitivität und Spezifität unterschieden werden kann, hat sich nicht halten können. Mit einem Verhältnis richtig-positiver zu richtig-negativer Aussage (Effizienz) von $80-85 \%(10,15)$ sind NPT-Meßergebnisse nur als einer von mehreren diagnostischen Bausteinen innerhalb eines Abklärungskonzeptes zu bewerten. Nur aufgrund der aufwendigen plethysmographischen NPT-Messung ist eine Einordnung der Patienten in organisch oder psychogen bedingt nicht zuverlässig möglich. Als Nachteil der Methode ist weiterhin zu werten, daß eine Differenzierung der organisch bedingten Ursachen und damit in vielen Fällen die gewünschte, therapierelevante Aussage nicht erreicht werden kann.

Die dopplersonographische Untersuchung der penilen Arterien stellt einen wesentlichen diagnostischen Schritt im multidisziplinären Abklärungsprogramm der erektilen Dysfunktion dar (19, 20). Sie dient zum Nachweis arteriosklerotischer Gefäßokklusionen, posttraumatischer Gefäßamputationen oder peniler Gefäßdysplasien. Sie stellt eine wesentliche Entscheidungshilfe für den Einsatz der Arteriographie dar, die vor revaskularisierenden operativen Maßnahmen unverzichtbar ist $(21,23)$.

Um eine quantifizierbare Aussage über die Arterialisation des Penis in flaccidem Zustand zu erhalten, wurden Messungen des penilen systolischen Blutdrucks eingeführt (11).
In Übereinstimmung mit eigenen Ergebnissen wird von Blaivas (1980) jedoch berichtet, daß einerseits ein erniedrigter PBI bei fehlender aorto-iliakaler Verschlußkrankheit, andererseits ein normaler PBI bei iliakalen Verschlüssen vorliegen kann (2). Diese Ergebnisse schränken den Wert der PBI-Messungen deutlich ein.

Über den Einsatz von Papaverin in Diagnostik und Therapie erektiler Dysfunktionen wurde erstmals von Virag (1982) berichtet (18).

Der Angriffspunkt des Papaverins, einem stark wirksamen Relaxans glatter Muskulatur, muß an den mikrovaskulären Regelstrukturen im Corpus cavernosum vermutet werden, insbesondere direkt an den mit glatter Muskulatur ausgekleideten kavernösen Hohlräumen. Eine Kombination von Papaverin und Phentolamin, einem Alpha-Rezeptorenblocker mit dadurch bedingter lokaler vasodilatatorischer Wirkung, wurde in Diagnostik und Therapie der erektilen Dysfunktion von Zorgniotti (25), Dhabuwala (8), Goldstein (12), Castillo (7) bisher angewandt.

Die Möglichkeit, durch die Injektion vasoaktiver Substanzen diagnostisch relevante Aussagen zu erzielen, sind in jüngeren Veröffentlichungen $(1,5,16,19,22)$ angesprochen. Aufgrund unserer eigenen Ergebnisse kommen wir zu folgenden Schlußfolgerungen:

1. Patienten mit gesundem Gefäßsystem (neurogen, psychogen) zeigen rasch einsetzende Erektionen (E 4 und $E$ 5) nach ca. 5-10 Minuten bei niedrig dosiertem Papaverin/PhentolaminGemisch. Mit einer durchschnittlichen Dosis von $0,43 \mathrm{ml}$ SKAT-Lösung (6,5 mg Papaverin/0,26 mg Phentolamin) wurde eine für die Kohabitation ausreichende Erektion erreicht. Alle Untersucher berichten über vereinzeltes Auftreten prolongierter Erektionen in dieser Gruppe.

2. Patienten mit vaskulär bedingter erektiler Dysfunktion durch pathologische inflow-Bedingungen (Arteriosklerose, Dysplasie oder posttraumatische Gefäßamputation) wiesen eine gegenüber der Gruppe 1 deutlich verzögert einsetzende Reaktion bei insgesamt verringerter Erektionsdauer auf. Die durchschnittlich zur Erzielung einer E 4/5 erforderlichen Dosis betrug $1,05 \mathrm{ml}$ SKAT-Lösung $(15,75 \mathrm{mg}$ Papaverin/ $0,52 \mathrm{mg}$ Phentolamin).

3. Patienten mit deutlicher venöser Insuffizienz erreichten auch bei einer Dosis von 3,0 ml (45 mg Papaverin/1,5 mg Phentolamin) keine $\mathrm{E} 4$ oder $\mathrm{E} 5$.

Unter Einbeziehung des geschilderten pharmakologischen Testverfahrens läßt sich eine therapieorientierte rationelle Diagnostik der erektilen Dysfunktion in Form eines Stufenplanes wie folgt standardisieren:

\begin{tabular}{cl} 
I Basisuntersuchungen: & $\begin{array}{l}\text { Anamnese, körperl. Unter- } \\
\text { suchung } \\
\text { Labor, Hormone } \\
\text { psychosex. Fragebogen } \\
\text { sonogr. Abdomen/Becken }\end{array}$ \\
& $\begin{array}{l}\text { Penile Doppler-Sonographie } \\
\text { SKAT-Testung }\end{array}$ \\
II Spezifische nicht-invasive & Sntersuchungen: \\
III Spezifische invasive Unter- & Kavernosographie \\
suchung (vor Op & Arteriographie \\
notwendig): & neurophysiol. Verfahren \\
\hline
\end{tabular}




\section{Literatur}

(1) Abber, J. C., T.F. Lue, R. D. McClure, R. D. Williams: Diagnostic Tests for Impotence: A Comparison of Papaverine Injections with the Penile Brachial Pressure Index and Nocturnal Penile Tumescence. J. Urol. 133, 4 (1985) $188 \mathrm{~A}$

(2) Blaivas, J. G., T. F. O'Donnell, P. Gottlieb, K. B. Labib: Comprehensive laboratory evaluation of impotent man. J. Urol. 124 (1980) 201-205

(3) Blum, M. D., R. R. Bahnson, T.N Porter, M. F. Carter: Effect of local alpha-adrenergic blockade on human penile erection. J. Urol. 134, 3 (1985) 479-481

(4) Brindley, G. S.: Cavernosal AlphaBlockade and Human Penile Erection. J. Physiol. 342 (1983) 4

(5) Brindley, G. S.: Cavernosal AlphaBlockade: A New Technique for Investigating and Treating Erectile Impotence. Br. J. Psychiatry 143, Oct.

(1983) 332-37

(6) Brindley, G. S.: New Treatment for Priapism. Lancet II (1984) 220-221

(7) Castillo, J., H. Y. Rodriguez, I. Z. Gutierrez, R. Cartagena: Intracavernous Injection of Two Vasoactive Drugs for Impotence: The Buffalo Experience. J. Urol. 133, 4 (1985) $190 \mathrm{~A}$

(8) Dhabuwala, C. B., P. Jaikishen, J. M. Pierce: The Effects of Intracavernosal Papaverine and Phentolamine on Penile Erections. J.Urol. 133, 4 (1985) $261 \mathrm{~A}$

(9) Farha, O. M. A., F. Hamound, M. Zohedey, M. El Garbawy, S. El Metet:
A Placebo Controlled Double Blind Study of the Effect of Intracavernous Injection of Phenoxybenzamine in the Diagnosis and Management of Organic Impotence. J. Urol. 133, 4 (1985) $220 \mathrm{~A}$

(10) Fisher, C. R., C. Schiavi, A. Edwards, D. M. Davis, M. Reitman, J. Fine: Evaluation of nocturnal penile tumescences in the differential diagnosis of sexual impotence. Arch. Gen. Psychiatry 36 (1979) 431-437

(11) Gaskell, P.: The importance of penile blood pressure in cases of impotence. CMA Jour 105 (1971) 10471051

(12) Goldstein, I., T. R. Payton, I. Saenz de Tejada, R. J. Krane: Pharmacologic Erections: Role in the Treatment of Neurologic Impotence. J. Urol. 133, 4 (1985) $261 \mathrm{~A}$

(13) Jevtich, M. J.: Non-invasive vascular and neurologic tests in use for evaluation of angiogenic impotence. Inter Angio 3 (1984) 225-232

(14) Juenemann, K. P., T. F. Lue, W. J. G. Hellstrom, G. R. Fournier, E. A. Tanagho: Hemodynamics of Papaverine and Phentolamine-Induced Penile Erection in Monkeys and Dogs. J. Urol. 133,4 (1985) $218 \mathrm{~A}$

(15) Karacan, J., P. J. Salis, R. L. Williams: The role of the sleep laboratory in the diagnosis and treatment of impotence. In: Williams, R. L., I. Karacan, S. H. Frazier (eds.) Sleep disorders, diagnosis and treatment. Wiley, New York (1978)
(16) Lue, T. F., H. Hricak, R. A. Tanangho, E. A.: Functional Evaluation of Penile Arterial Blood Flow During Erection. J. Urol. 133, 4 (1985) 218 A (17) Lue, T. F., H. Hricak, K. W. Marich, E. A. Tanangho: Evaluation of arteriogenic impotence with intracorporeal injection of papaverine and the duplex ultrasound scanner. Semin.

Urol. 3, 1 (1985) 43-48

(18) Virag, R.: Intracavernous Injection of Papaverine for Erectile Failure. Lancet II, Oct. 23 (1982) 938

(19) Virag, R., D. Frydman, M. Legman, $H$. Virag: Intracavernous Injection of Papaverine as a Diagnostic and Therapeutic Method in Erectile Failure. Angiology 35,2 (1984) 79-87

(20) Virag, R., D. Frydman, M. Legman, J. Floresco, P. Bouilly: Hemody namic evaluation of arterial and venous lesions as a cause of impotence. Inter Angio 3 (1984) 241-246

Dr. med. W. Bäbren

Arzt für Radiologie

Bundeswehrkrankenhaus Ulm

Oberer Eselsberg 40

D-7900 Ulm
(21) Virag, R.: Conservative medical and surgical treatment of impotence. Symposium: Controversy in the diagnosis and treatment of erectile impotence. Leiden 1984

(22) Virag, R.: Human Penile Erection: An Extensive Study of the Effects of Vasoactive Compounds on the Cavernous Tissue and the Penile Arteries. J. Urol. 133,4 (1985) 190 A

(23) Zorgniotti, A. W., G. Padula, W. W. Shaw: Selective arterio graphy for vascular impotence. World J. Urol. 1 (1983) 213-217

(24) Zorgniotti, A. W.: Self Administered Intracavernous Injection of a Vasoactive Drug for Impotence in Diabetics. J. Urol. 131, 4 (1984) $201 \mathrm{~A}$

(25) Zorgniotti, A. W., R. S. Lefleur: Auto-Injection of the Corpus Cavernosum with a Vasoactive Drug Combination for Vasculogenic Impotence. J. Urol. 133, 1 (1985) 39-41 